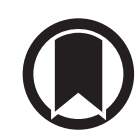

CrossMark

\section{EmPHasis-10 health-related quality of life score predicts outcomes in patients with idiopathic and connective tissue disease- associated pulmonary arterial hypertension: results from a UK multicentre study}

Robert A. Lewis ${ }^{1,2}$, lain Armstrong ${ }^{1}$, Carmel Bergbaum ${ }^{3}$, Melanie J. Brewis ${ }^{4}$, John Cannon ${ }^{5}$, Athanasios Charalampopoulos ${ }^{1}$, A. Colin Church ${ }^{4}$, J. Gerry Coghlan ${ }^{6}$, Rachel J. Davies ${ }^{7}$, Konstantinos Dimopoulos $\mathbb{1}^{3}$, Charlie Elliot ${ }^{1}$, J. Simon R. Gibbs ${ }^{7}$, Wendy Gin-Sing ${ }^{7}$, Gulam Haji Abdul G. Hameed (10 ${ }^{1,2}$, Luke S. Howard ${ }^{7}$, Martin K. Johnson ${ }^{4}$, Aleksander Kempny ${ }^{3}$, David G. Kiely (1) ${ }^{1,2}$, Francesco Lo Giudice ${ }^{7}$, Colm McCabe ${ }^{3}$, Andrew J. Peacock ${ }^{4}$, Oyinkansola Peleyeju ${ }^{6}$, Joanna Pepke-Zaba ${ }^{5}$, Gary Polwarth $\mathbb{1}^{5}$, Laura Price $0^{3}$, Ian Sabroe ${ }^{1,2}$, Benjamin E. Schreiber ${ }^{6}$, Karen Sheares ${ }^{5}$, Dolores Taboada ${ }^{5}$, A.A. Roger Thompson (10) ${ }^{1,2}$, Mark R. Toshner (1) ${ }^{5}$, Ivy Wanjiku', S. John Wort ${ }^{3}$, Janelle Yorke ${ }^{8}$ and Robin Condliffe $\mathbb{B}^{1}$

@ERSpublications

The emPHasis-10 health-related quality of life score is an independent predictor of mortality in idiopathic and connective tissue disease-related pulmonary arterial hypertension, and has utility in risk stratification. https://bit.ly/2MrLFLn

Cite this article as: Lewis RA, Armstrong I, Bergbaum C, et al. EmPHasis-10 health-related quality of life score predicts outcomes in patients with idiopathic and connective tissue disease-associated pulmonary arterial hypertension: results from a UK multicentre study. Eur Respir J 2021; 57: 2000124 [https://doi.org/ $10.1183 / 13993003.00124-2020]$.

ABSTRACT Health-related quality of life (HRQoL) scores assess symptom burden in pulmonary arterial hypertension $(\mathrm{PAH})$ but data regarding their role in prognostication and risk stratification are limited. We assessed these relationships using the emPHasis-10 HRQoL measure.

1745 patients with idiopathic PAH (IPAH), drug-induced PAH (DPAH), heritable PAH (HPAH) (collectively "(I/D/H)PAH"), or connective tissue disease-associated PAH (CTD-PAH), who had completed emPHasis-10 questionnaires at one of six UK referral centres between 2014 and 2017, were identified. Correlations with exercise capacity and World Health Organization (WHO) functional class were assessed, and exploratory risk stratification thresholds were tested.

Moderate correlations were seen between emPHasis-10 scores and 6-min walk distance $(\mathrm{r}=-0.546)$, incremental shuttle walk distance $(\mathrm{r}=-0.504)$ and WHO functional class $(\mathrm{r}=0.497)$ (all $\mathrm{p}<0.0001)$. Distribution of emPHasis-10 score differed significantly between each WHO functional class (all $\mathrm{p}<0.0001$ ). On multivariate analysis, emPHasis-10 score, but not WHO functional class, was an independent predictor of mortality. In a risk stratification approach, scores of 0-16, 17-33 and 34-50 identified incident patients with 1 -year mortality of $5 \%, 10 \%$ and $23 \%$, respectively. Survival of patients in WHO functional class III could be further stratified using an emPHasis-10 score $\geqslant 34(\mathrm{p}<0.01)$. At follow-up, patients with improved emPHasis-10 scores had improved exercise capacity $(p<0.0001)$ and patients who transitioned between risk groups demonstrated similar survival to patients originally in those risk groups.

The emPHasis-10 score is an independent prognostic marker in patients with (I/D/H)PAH or CTDPAH. It has utility in risk stratification in addition to currently used parameters. Improvement in emPHasis-10 score is associated with improved exercise capacity.

This article has an editorial commentary: https://doi.org/10.1183/13993003.04376-2020

Received: 21 Jan 2020 | Accepted: 28 May 2020

Copyright OERS 2021. This article is open access and distributed under the terms of the Creative Commons Attribution Licence 4.0. 


\section{Introduction}

Pulmonary arterial hypertension (PAH) is a rare condition, characterised by increased pulmonary vascular resistance (PVR) and progressive right-ventricular failure leading to premature death [1]. Exertional breathlessness and limitation in physical activity are typically the earliest reported symptoms and may be caused by a number of mechanisms $[2,3]$. Exercise limitation may be objectively assessed by exercise testing, but limitations of day-to-day physical activity are typically assessed by healthcare professionals using the World Health Organisation (WHO) functional class system.

The importance of assessing patient-reported outcome measures (PROMs) in patients with pulmonary hypertension $(\mathrm{PH})$ is now recognised $[4,5]$ and three $\mathrm{PH}$-specific tools for assessing health-related quality of life (HRQoL) have been developed [6-8]. One of these tools, emPHasis-10, is comprised of 10 fields and results in a score out of 50 where a higher score represents a higher symptom burden. It can be completed quickly by patients and is free to use, and so is well-suited to routine clinical use [7]. The emPHasis-10 score was found to correlate strongly with measures of HRQoL, breathlessness and psychological morbidity, and has high test-retest and internal consistency [7]. In addition, the emPHasis-10 questionnaire has been translated into a number of other languages [9, 10]. A previous single-centre study of emPHasis-10 in patients with $\mathrm{PAH}$ (predominantly associated with congenital heart disease) and chronic thromboembolic pulmonary hypertension (CTEPH) demonstrated prognostic significance and a correlation with WHO functional class [11]. Although risk stratification has an established central role in the management of patients with PAH, PROMs are not incorporated in current risk assessment tools [12-14].

Routine HRQoL assessment using a PH-specific tool has been a mandatory field in the UK National Audit of Pulmonary Hypertension since 2014 [15]. We performed a multi-centre study on a large cohort of patients with idiopathic PAH (IPAH), drug-induced PAH (DPAH), heritable PAH (HPAH) (collectively "(I/D/H)PAH") and connective tissue disease-associated PAH (CTD-PAH), to further assess the relationship between emPHasis-10 score and mortality, to identify correlations with clinical parameters (including exercise capacity) and to determine whether a threshold approach for risk stratification could be applied.

\section{Methods}

Local databases for six out of the seven PH-referral centres in the UK, which together manage $94 \%$ of adult patients with a diagnosis of $\mathrm{PAH}$, were interrogated [15]. Patients with $\mathrm{PAH}$ were diagnosed as per contemporaneous international guidelines (mean pulmonary arterial pressure (PAP) $\geqslant 25 \mathrm{mmHg}$ and pulmonary arterial wedge pressure (PAWP) $\leqslant 15 \mathrm{mmHg}$ in the absence of thromboembolic disease or conditions associated with other forms of PH) [16]. Anonymised demographic, haemodynamic, spirometric, exercise, emPHasis-10 and mortality data were retrieved for all patients with a diagnosis of IPAH, DPAH, HPAH (hereafter grouped as “(I/D/H)PAH”), or CTD-PAH with at least one recorded emPHasis-10 score between January 01, 2014 and May 31, 2018. Incident patients were required to have an emPHasis-10 score at the point of diagnosis, which was possible if diagnosed from 2014 onwards since its clinical use was introduced in the UK during that year. For prevalent patients (i.e. those diagnosed prior to 2014 or for whom no emPHasis-10 score was available at the time of diagnosis), the first available emPHasis-10 score was used. In either group, the first emPHasis-10 score was described as the baseline measurement. All patients were under regular clinical follow-up and the outcome measured was death or transplant by May 31, 2019. Follow-up data were retrieved for the first visit between 3 and 12 months after baseline emPHasis-10 score was measured.

\section{Statistical analysis}

Statistical analysis was performed using SPSS Statistics version 26 (IBM Inc, Armonk, NY, USA) and GraphPad Prism version 8 (GraphPad Spftware, San Diego, CA, USA). Continuous data were displayed as either mean \pm standard deviation (SD) or median (1st-3rd quartile) for non-parametric data. Demographics were compared using paired and unpaired T-tests for parametric data and Wilcoxon signed-rank and

Affiliations: 'Sheffield Pulmonary Vascular Disease Unit, Royal Hallamshire Hospital, Sheffield, UK. ${ }^{2}$ Dept of Infection, Immunity and Cardiovascular Disease, University of Sheffield, Sheffield, UK. ${ }^{3}$ National Pulmonary Hypertension Service, Royal Brompton Hospital and Imperial College, London, UK. ${ }^{4}$ Scottish Pulmonary Vascular Unit, Golden Jubilee National Hospital, Glasgow, UK. ${ }^{5}$ Pulmonary Vascular Disease Unit, Royal Papworth Hospital, Cambridge, UK. ${ }^{6}$ Pulmonary Hypertension Unit, Royal Free Hospital, London, UK.

${ }^{7}$ National Pulmonary Hypertension Service, Hammersmith Hospital, London, UK. ${ }^{8}$ School of Nursing, Midwifery and Social Work, University of Manchester, Manchester, UK.

Correspondence: Robin Condliffe, Sheffield Pulmonary Vascular Disease Unit, Royal Hallamshire Hospital, Sheffield S10 2JF, UK. E-mail: robin.condliffelanhs.net 
Mann-Whitney U-tests for non-parametric data. Frequencies were compared using Chi-squared. For Cox regression modelling, parameters of known prognostic significance in PAH were utilised (age, gender, presence of CTD (rather than (I/D/H)PAH), mean right atrial pressure (RAP), cardiac index and walking distance. Collinearity was assessed by measuring the variance inflation factor and tolerance between variables. EmPHasis-10 score was entered as a continuous variable in the multivariable model. Multivariate Cox regression analysis was performed in a forward direction on all parameters with a p-value of less than 0.2 on univariate analysis. Data were scaled to the mean and hazard ratios were based on the z-score. Two types of walking test were used (the 6-min walk test (6MWT) and the incremental shuttle walk test (ISWT)) and so, for multivariate modelling, distances were converted to a z-score and combined as a single variable. For all statistical tests other than multivariate analysis, a p-value of less than 0.05 was considered significant. Kaplan-Meier survival curves were compared using log-rank Chi-squared and were truncated at 4 years based on the census date. Correlations were assessed using either Pearson or Spearman rank, as appropriate. Risk models were compared using the c-statistic identified from receiver operating characteristic (ROC) curve analysis. The minimal detectable change (MDC) for emPHasis-10 score was calculated using the formula: $\mathrm{MDC}=1.96 \cdot \sqrt{ } 2 \cdot$ standard error ( $\mathrm{SE}$ ) of measurement [17]. Ethical approval was granted (IRAS 254446).

\section{Results}

A total of 1745 patients with (I/D/H)PAH ( $\mathrm{n}=994)$ or CTD-PAH $(\mathrm{n}=751)$ who had at least one recorded emPHasis-10 score were identified. There was a female predominance $(73 \%)$ and $35 \%$ of patients were incident and treatment-naïve at the time of baseline emPHasis-10 score measurement. The emPHasis-10 score was higher in patients with CTD-PAH (median 30 (19-38)) than in patients with (I/D/H)PAH (median $28(17-37))(\mathrm{p}=0.001)$. Baseline demographics are displayed in table 1.

\section{Correlation with clinical parameters}

Moderate correlations (all $\mathrm{p}<0.0001$ ) were seen between baseline emPHasis-10 score and WHO functional class $(\mathrm{r}=0.50)$, 6-min walk distance (6MWD) $(\mathrm{r}=-0.55)$ and incremental shuttle walk distance (ISWD) $(\mathrm{r}=-0.50)$, as illustrated in table 2 . In incident patients with right-heart catheter data available $(\mathrm{n}=591)$,

\section{TABLE 1 Patient characteristics}

\begin{tabular}{|c|c|c|c|c|}
\hline & All (1745) & (I/D/H)PAH (n=994) & CTD-PAH (n=751) & p-value \\
\hline Female sex $\%$ & 73 & 66 & 82 & $<0.0001$ \\
\hline Age at diagnosis years & $59 \pm 17$ & $55 \pm 18$ & $64 \pm 13$ & $<0.0001$ \\
\hline Incident patients \% ( $n=618$ ) & 35 & 29 & 44 & $<0.0001$ \\
\hline $\mathrm{FEV}_{1} \%$ predicted ( $\mathrm{n}=1457$ ) & $82 \pm 21$ & $84 \pm 19$ & $80 \pm 23$ & 0.0001 \\
\hline FVC $\%$ predicted ( $n=1459)$ & $92 \pm 23$ & $95 \pm 20$ & $89 \pm 27$ & $<0.0001$ \\
\hline $\mathrm{FEV}_{1} / \mathrm{FVC}(\mathrm{n}=1459)$ & $73 \pm 13$ & $74 \pm 13$ & $73 \pm 14$ & 0.078 \\
\hline Mean RAP mmHg ( $=1503$ ) & $9 \pm 6$ & $10 \pm 6$ & $8 \pm 5$ & $<0.0001$ \\
\hline Mean PAP mmHg(n=1573) & $48 \pm 13$ & $53 \pm 13$ & $41 \pm 11$ & $<0.0001$ \\
\hline PAWP $\mathrm{mmHg}(\mathrm{n}=1496)$ & $9 \pm 4$ & $9 \pm 4$ & $9 \pm 3$ & 0.56 \\
\hline PVR Wood units ( $n=1378)$ & $10.5 \pm 5.8$ & $12.0 \pm 5.7$ & $8.7 \pm 5.4$ & 0.0001 \\
\hline Cardiac output L- $\min ^{-1}(n=1465)$ & $4.2 \pm 1.5$ & $4.0 \pm 1.5$ & $4.3 \pm 1.5$ & $<0.0005$ \\
\hline Cardiac index $L \cdot \mathrm{min}^{-1} \cdot \mathrm{m}^{-2}(\mathrm{n}=1305)$ & $2.4 \pm 0.8$ & $2.2 \pm 0.8$ & $2.5 \pm 0.8$ & $<0.0001$ \\
\hline EmPHasis-10 score & $29(18-38)$ & $28(17-37)$ & $30(19-38)$ & 0.001 \\
\hline \multicolumn{5}{|l|}{ WHO functional class ${ }^{\#} \%$ ( $\left.n=1725\right)$} \\
\hline Class I & 3 & 4 & 1 & \\
\hline Class II & 23 & 26 & 20 & \\
\hline Class III & 61 & 57 & 67 & \\
\hline Class IV & 13 & 13 & 12 & \\
\hline $6 M W D^{*}(n=659)$ & $310(180-408)$ & 340 (192-432) & $241(141-360)$ & $<0.0001$ \\
\hline ISWD $^{*}(\mathrm{n}=797)$ & $150(70-270)$ & $160(80-350)$ & $140(60-228)$ & 0.001 \\
\hline \multicolumn{5}{|c|}{$\begin{array}{l}\text { Data are presented as } n \text {, mean } \pm \text { SD, or median (1st-3rd quartile). PAH: pulmonary arterial hypertension; } \\
\text { CTD: connective tissue disease; IPAH: idiopathic PAH; DPAH: drug-induced PAH; HPAH: heritable PAH; } \\
\text { CTD-PAH: CTD-associated PAH; FEV } \text { : forced expiratory volume in } 1 \mathrm{~s} \text {; FVC: forced vital capacity; RAP: } \\
\text { right atrial pressure; PAP: pulmonary arterial pressure; PAWP: pulmonary arterial wedge pressure; PVR: } \\
\text { pulmonary vascular resistance; WHO: World Health Organization; } 6 \mathrm{MWD} \text { : } 6 \text {-min walk distance; ISWD: } \\
\text { incremental shuttle walk distance; SD: standard deviation. \#: variables were recorded at the time of } \\
\text { baseline emPHasis-10 measurement lother variables were recorded at diagnosis). Baseline } 6 \mathrm{MWD} \text { and } \\
\text { ISWD were available in } 38 \% \text { and } 46 \% \text { of patients, respectively, with no overlap. }\end{array}$} \\
\hline
\end{tabular}


TABLE 2 Correlation of emPHasis-10 score and World Health Organization (WHO) functional class with walk distance and pulmonary haemodynamics

\begin{tabular}{|c|c|c|c|c|c|}
\hline & 6MWD m & ISWD m & Mean RAP mmHg & Cardiac index $\mathrm{L} \cdot \mathrm{min}^{-1} \cdot \mathrm{m}^{-2}$ & PVR Wood units \\
\hline
\end{tabular}

Correlation was assessed by Pearson or Spearman-rank tests as appropriate. 6MWD: 6-min walk distance; ISWD: incremental shuttle walk distance; RAP: right atrial pressure; PVR: pulmonary vascular resistance. ${ }^{\#}: \mathrm{p}<0.001$.

there were weak correlations with mean RAP ( $\mathrm{r}=0.21)$, cardiac index $(\mathrm{r}=-0.21)$ and PVR ( $\mathrm{r}=0.17)$ (all $\mathrm{p}<0.0001)$. Correlations were similar in the subgroups of $(\mathrm{I} / \mathrm{D} / \mathrm{H}) \mathrm{PAH}$ and CTD-PAH, apart from PVR where correlation was significant in CTD-PAH $(r=0.21 ; \mathrm{p}<0.0005)$ but not in $(\mathrm{I} / \mathrm{D} / \mathrm{H}) \mathrm{PAH}(\mathrm{r}=0.11 ; \mathrm{p}=0.8)$. Correlations between WHO functional class, walk distance and haemodynamics are also shown in table 2 .

Distribution of emPHasis-10 score by WHO functional class at baseline is shown in figure 1. Median emPHasis-10 scores were 3, 19, 31 and 40 in WHO functional classes I, II, III and IV, respectively, with highly significant differences between the scores in each functional class (all $\mathrm{p}<0.0001$ ).

\section{Risk stratification}

During the course of the study 674 patients (39\%) died, of which 240 (14\%) died within 1 year of baseline emPHasis-10 score measurement. The 1-year mortality in incident and prevalent patients was $16 \%$ and $12 \%$, respectively. An exploratory three-level score was developed based on a tertile group approach: scores of $0-16$ were defined as low-risk, $17-33$ as intermediate-risk and $34-50$ as high-risk. Using these thresholds, $22 \%$ of all patients were defined as low-risk for 1 -year mortality, $41 \%$ as intermediate-risk and $37 \%$ as high-risk. Survival curves for these risk groups are shown for incident patients in figure $2 \mathrm{a}$, for prevalent patients in figure $2 \mathrm{~b}$ and for all patients in figure $2 \mathrm{c}$. In incident patients, 1-year mortality for the low, intermediate and high-risk groups was $5 \%, 10 \%$ and $23 \%$, respectively. In prevalent patients it was $4 \%, 13 \%$ and $20 \%$, respectively, while for all patients it was $4 \%, 12 \%$ and $21 \%$, respectively. In all patients with $(\mathrm{I} / \mathrm{D} / \mathrm{H}) \mathrm{PAH}, 1$-year mortality for the low, intermediate and high-risk groups was $4 \%, 9 \%$ and $18 \%$, respectively, whereas in CTD-PAH it was $6 \%, 15 \%$ and $25 \%$. Incident patients in functional class III who were in the low/intermediate emPHasis-10 risk group (emPHasis-10 score 0-33) had superior survival than those in the high-risk group (emPHasis-10 score 34-50), with 1 -year survival of $90 \%$ versus $81 \%$ and 3 -year survival of $67 \%$ versus $56 \%(\mathrm{p}<0.01)$ (figure 3 ). Very similar observations were made in functional class III patients at their first follow-up visit.

\section{Survival analysis}

Three multivariate analysis models were developed in the incident population (table 3). Model 1 utilised accepted prognostic parameters (age, gender, CTD-PAH rather than (I/D/H)PAH, WHO functional class, mean RAP and cardiac index). EmPHasis-10 and exercise capacity were sequentially added into models 2

FIGURE 1 Distribution of emPHasis10 score by World Health Organization (WHO) functional class at baseline

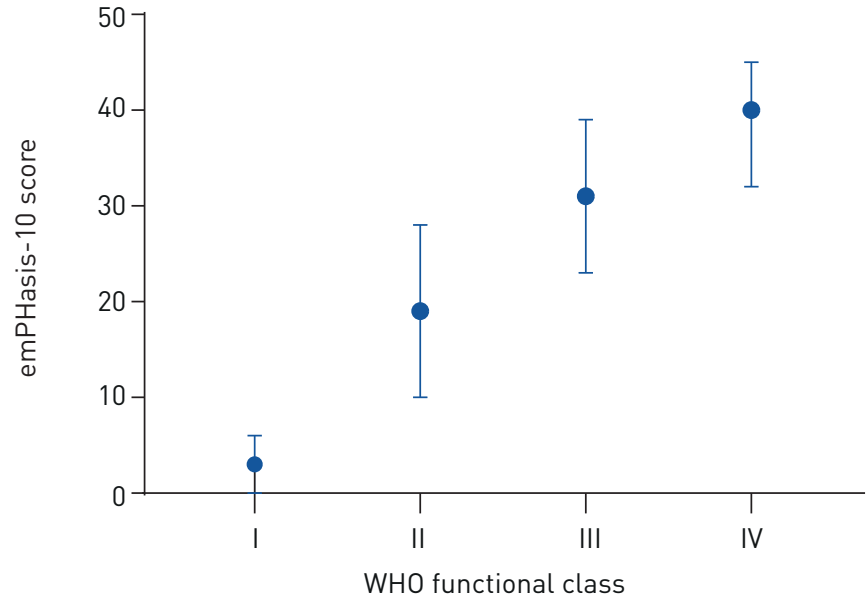




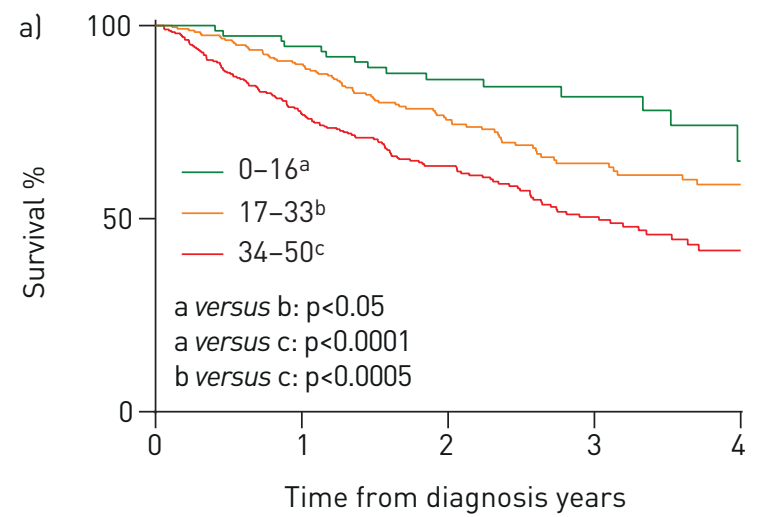

$\begin{array}{cccccc}- & 75 & 71 & 50 & 29 & 6 \\ - & 240 & 216 & 126 & 68 & 36 \\ -303 & 234 & 136 & 69 & 22\end{array}$

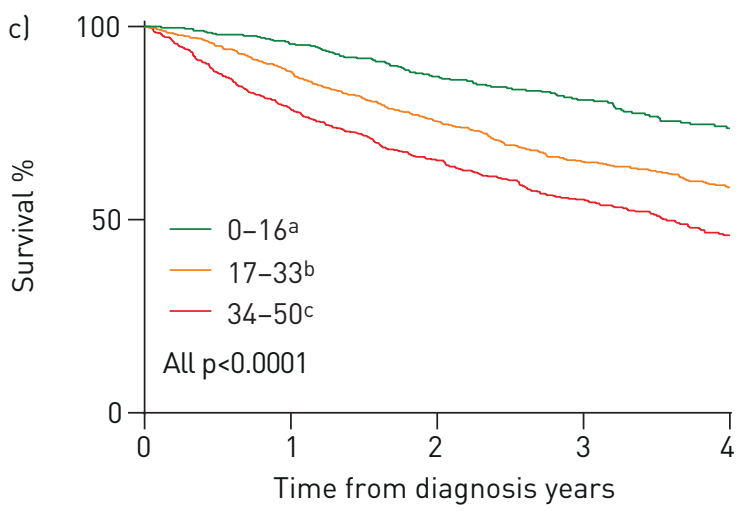

$\begin{array}{rllll}379 & 362 & 300 & 229 & 147 \\ -713 & 630 & 460 & 316 & 212 \\ -\quad 653 & 514 & 362 & 232 & 130\end{array}$

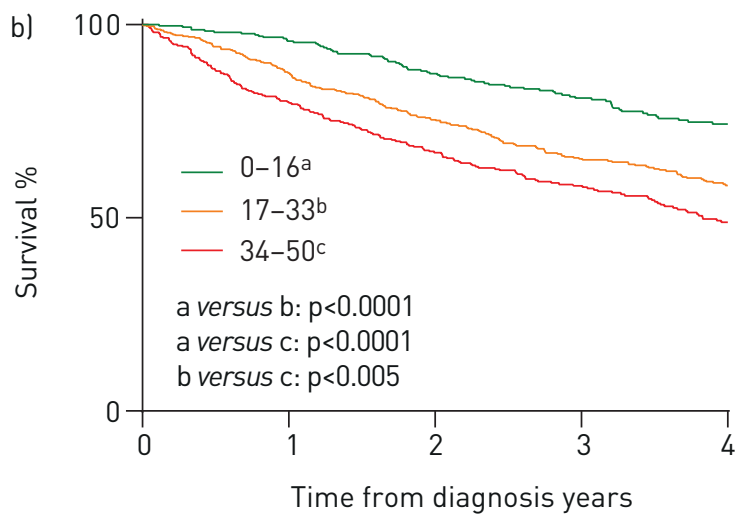

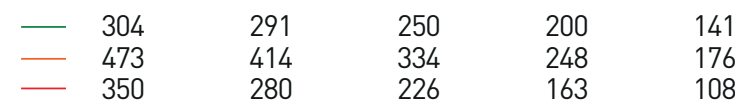

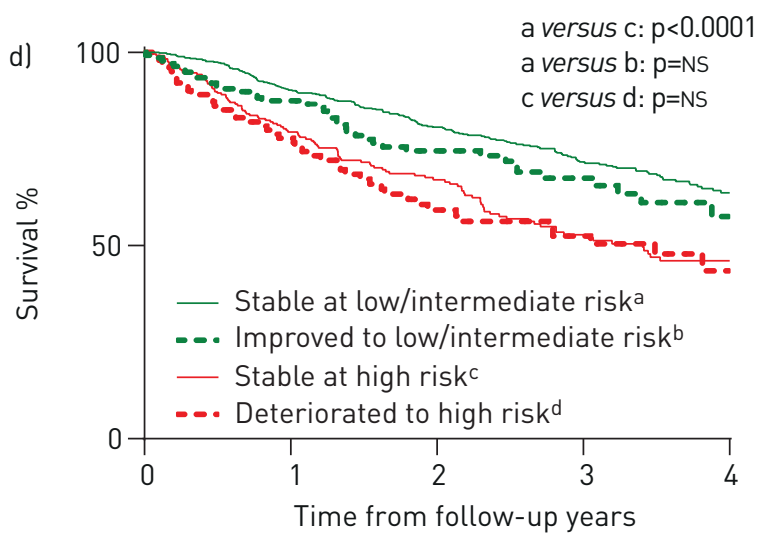

$\begin{array}{cccccc}- & 565 & 483 & 360 & 237 & 101 \\ - & 139 & 103 & 65 & 35 & 15 \\ - & 247 & 178 & 122 & 70 & 24 \\ -= & 101 & 70 & 41 & 26 & 9\end{array}$

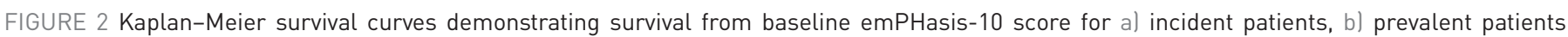
and c) all patients, as well as d) risk transition in all patients between baseline and follow-up emPHasis-10 score. Ns: not significant.

and 3. Unlike WHO functional class, emPHasis-10 score was an independent predictor of outcome in model 2 (scaled hazard ratio $1.565, \mathrm{p}<0.0001$ ) and model 3 (scaled hazard ratio $1.226, \mathrm{p}<0.05$ ). There was no significant collinearity between parameters used in the model.

\section{Magnitude of change}

The MDC for emPHasis-10 score was calculated to be nine. Follow-up emPHasis-10 data were available for 1068 patients (61\%). EmPHasis-10 score changed by at least nine points between baseline and follow-up in $33 \%$ of patients $(32 \%$ for $(\mathrm{I} / \mathrm{D} / \mathrm{H}) \mathrm{PAH}$ and $34 \%$ for CTD-PAH). Thirty-seven percent of patients moved risk groups, of which $19 \%$ improved by at least one risk group. In patients who moved from high risk to intermediate or low risk, the median change in emPHasis-10 score was -12 points ( -6 to -19 points) and in patients who deteriorated to high-risk the median change was +13 points $(+8$ to +17 points). Patients who either improved to low or intermediate-risk, or deteriorated to high-risk, demonstrated similar long-term survival to patients originally in those risk groups (figure $2 \mathrm{~d}$ ).

On paired testing in patients with a follow-up emPHasis-10 score, those who improved emPHasis-10 score by the MDC of nine points or greater had significantly improved walk distances at follow-up. ISWD increased by $30 \mathrm{~m}(0-90 \mathrm{~m})(\mathrm{p}<0.0001)$ (figure $4 \mathrm{a})$ while 6MWD increased by a median distance of $32 \mathrm{~m}$ $(-4$ to $113 \mathrm{~m})(\mathrm{p}<0.005)$ (figure $4 \mathrm{~b})$. A significant fall in ISWD of $-20 \mathrm{~m}(-60$ to $0 \mathrm{~m})(\mathrm{p}<0.0001)$ (figure 4a) and no significant change in 6 MWD $(0 \mathrm{~m},-29$ to $57 \mathrm{~m}$ ) (figure $4 \mathrm{~b}$ ) was observed in patients whose 
FIGURE 3 Survival in incident patients with World Health Organization (WHO) functional class III symptoms, stratified by emPHasis10 score $(\leqslant 33$ or $\geqslant 34)$.

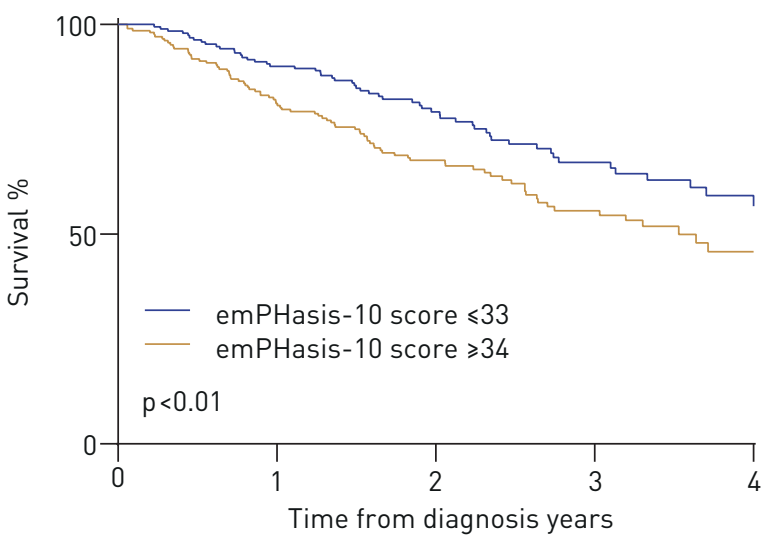

$\begin{array}{rrrrr}-191 & 172 & 101 & 53 & 23 \\ -\quad 207 & 168 & 101 & 53 & 16\end{array}$

emPHasis-10 score deteriorated by at least nine points. In the remaining patients, in whom there was a change of less than nine points, there was no significant change in either ISWD ( $0 \mathrm{~m},-30$ to $20 \mathrm{~m})$ or 6MWD $(0 \mathrm{~m},-11$ to $53 \mathrm{~m})$. The relationship between the change in emPHasis-10 score and the change in walk distance differed depending on whether patients were incident or prevalent at the time of their

\section{TABLE 3 Univariate and multivariate analysis in incident patients}

\begin{tabular}{|c|c|c|c|c|}
\hline \multirow[t]{2}{*}{ Model 1} & \multicolumn{2}{|c|}{ Univariate } & \multicolumn{2}{|c|}{ Multivariate } \\
\hline & $\begin{array}{l}\text { Scaled hazard } \\
\text { ratio }\end{array}$ & p-value & $\begin{array}{l}\text { Scaled hazard } \\
\text { ratio }\end{array}$ & p-value \\
\hline \multicolumn{5}{|l|}{ Model 1} \\
\hline Age & 2.063 & $<0.0001$ & 2.177 & $<0.0001$ \\
\hline Gender (ref. female) & 1.316 & 0.054 & & \\
\hline CTD-PAH (ref. (I/D/H)PAH) & 1.336 & 0.031 & 1.444 & 0.017 \\
\hline WHO functional class III (ref. class I and II) & 1.817 & 0.009 & & \\
\hline WHO functional class IV (ref. class I and II) & 3.642 & $<0.0001$ & 2.978 & $<0.0001$ \\
\hline Mean RAP & 1.196 & 0.006 & 1.227 & 0.005 \\
\hline Cardiac index & 0.756 & 0.001 & & \\
\hline \multicolumn{5}{|l|}{ Model 2} \\
\hline Age & 2.063 & $<0.0001$ & 2.180 & $<0.0001$ \\
\hline Gender (ref. female) & 1.316 & 0.054 & & \\
\hline CTD-PAH (ref. (I/D/H)PAH) & 1.336 & 0.031 & & \\
\hline WHO functional class III (ref. class I and II) & 1.817 & 0.009 & & \\
\hline WHO functional class IV (ref. class I and II) & 3.642 & $<0.0001$ & & \\
\hline Mean RAP & 1.196 & 0.006 & & \\
\hline Cardiac index & 0.756 & 0.001 & & \\
\hline EmPHasis-10 score & 1.518 & $<0.0001$ & 1.447 & $<0.0001$ \\
\hline \multicolumn{5}{|l|}{ Model 3} \\
\hline Age & 2.063 & $<0.0001$ & 1.860 & $<0.0001$ \\
\hline Gender (ref. female) & 1.316 & 0.054 & & \\
\hline CTD-PAH (ref. (I/D/H)PAH) & 1.336 & 0.031 & & \\
\hline WHO functional class III (ref. class I and II) & 1.817 & 0.009 & & \\
\hline WHO functional class IV (ref. class I and II) & 3.642 & $<0.0001$ & & \\
\hline Mean RAP & 1.196 & 0.006 & & \\
\hline Cardiac index & 0.756 & 0.001 & & \\
\hline EmPHasis-10 score & 1.518 & $<0.0001$ & 1.226 & 0.047 \\
\hline Walk distance ${ }^{\#}$ & 0.461 & $<0.0001$ & 0.574 & $<0.0001$ \\
\hline \multicolumn{5}{|c|}{$\begin{array}{l}\text { PAH: pulmonary arterial hypertension; CTD: connective tissue disease; IPAH: idiopathic PAH; DPAH: } \\
\text { drug-induced PAH; HPAH: heritable PAH; CTD-PAH: CTD-associated PAH; WHO: World Health } \\
\text { Organization; RAP: right-atrial pressure. \#: two types of walking test were used, the 6-min walk test } \\
\text { (6MWT) and the incremental shuttle walk test (ISWT). For Cox regression modelling, distances were } \\
\text { converted to a z-score and combined. }\end{array}$} \\
\hline
\end{tabular}



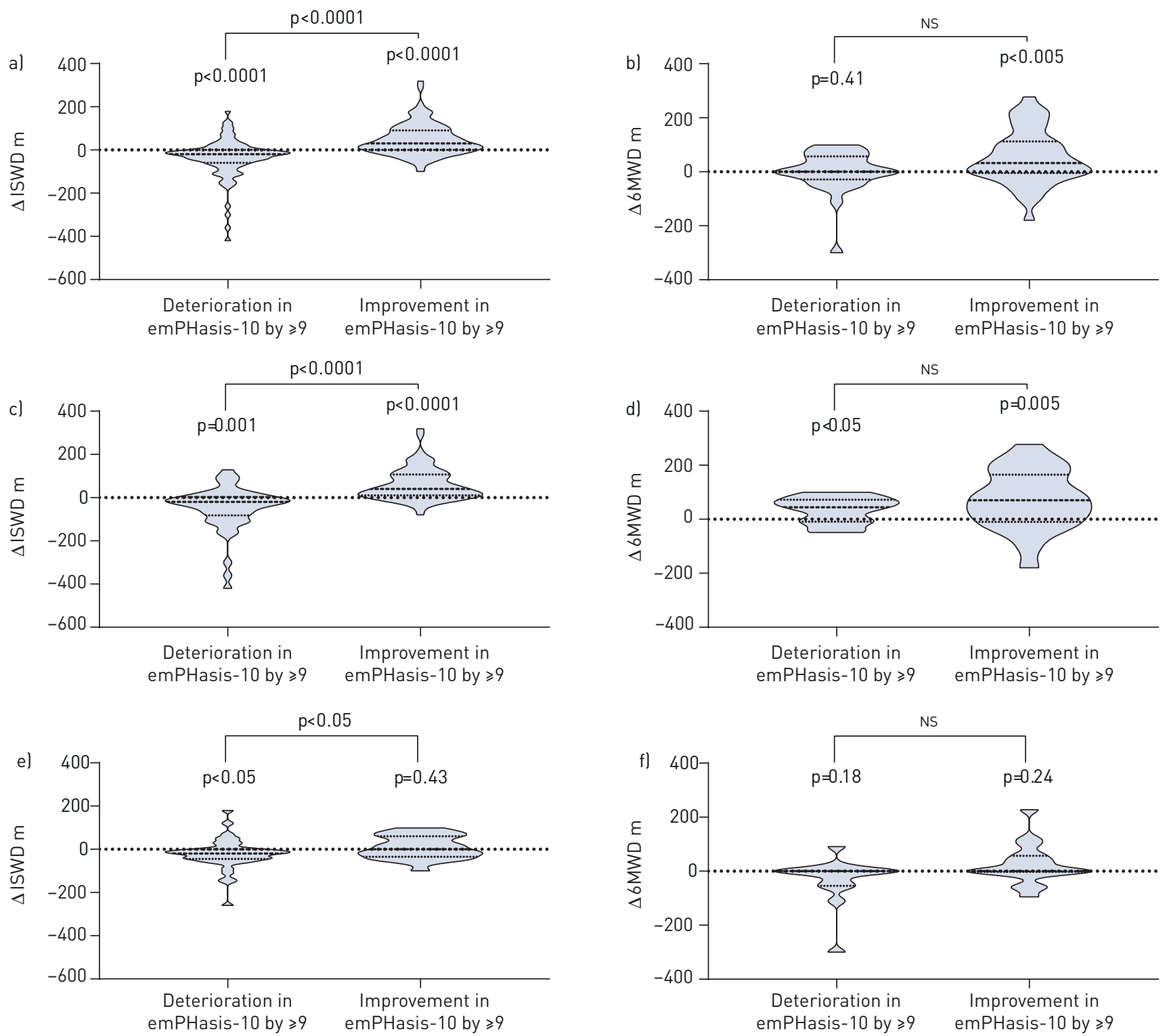

FIGURE 4 Change, as illustrated by violin plots (where: dashed line=median; dotted lines=25th and 75th percentiles), in incremental shuttle walk distance (ISWD) and 6-min walk distance (6MWD) in patients whose emPHasis-10 score deteriorated by $\geqslant 9$ or improved by $\geqslant 9$ between baseline and follow-up (la and b) all patients, (c and d) incident patients and (e and f) prevalent patients).

baseline walk (the relationship being stronger in incident patients) and whether they performed the ISWT or 6MWT (the relationship being stronger in patients who performed the ISWT). In patients whose emPHasis-10 score deteriorated by nine or more points, ISWD fell significantly in both the incident and prevalent populations. In patients whose emPHasis-10 score improved by nine or more points, ISWD increased significantly in incident but not prevalent, patients (figures $4 \mathrm{c}$ and $4 \mathrm{e}$ ). In patients for whom a 6MWT was performed, an improvement was observed in incident patients whose emPHasis-10 score either improved or deteriorated by nine or more points (figure $4 \mathrm{~d}$ ), while no significant change was seen in prevalent patients (figure 4f).

\section{Discussion}

To our knowledge, this is the largest study to assess the role of quality of life (QoL) scores in patients with PAH. In this multi-centre study we report on data from centres treating the vast majority of the adult PAH population in the UK. We have demonstrated that the emPHasis-10 score is an independent predictor of outcomes when adjusting for haemodynamics and WHO functional class, and also that it has utility in risk stratification, including within patients in WHO functional class III. We have also observed moderate correlations with WHO functional class and exercise capacity, and weaker correlations with 
pulmonary haemodynamics. Furthermore, we have also demonstrated that improvement in emPHasis-10 score, as opposed to a static or worsening score, is associated with improvements in exercise capacity.

Generic (e.g. the 36-item Short Form Health Survey (SF-36)), heart-failure specific (e.g. the Minnesota Living with Heart Failure Questionnaire (MLHFQ)) and PH-specific (e.g. emPHasis-10 and the Cambridge Pulmonary Hypertension Outcome Review (CAMPHOR)) PROMs have previously been identified as having prognostic importance in PAH [11, 18-20]. Correlations between CAMPHOR and both SF-36 and $6 \mathrm{MWD}$ have also been demonstrated $[19,21,22]$. The widespread clinical use of the CAMPHOR score may, however, be limited by its length (65 fields over three domains: symptoms, functioning and QoL) and lack of open access [23]. A third PH-specific PROM, the Pulmonary Arterial Hypertension-Symptoms and Impact (PAH-SYMPACT) tool, which consists of 22 fields over two domains: symptoms and impacts, has also been developed [8]. Although PAH-SYMPACT is responsive to change, its relationship to haemodynamics and survival is not known [24].

A previous single-centre study involving 687 patients (congenital heart disease-associated PAH $(n=314)$, $(\mathrm{I} / \mathrm{D} / \mathrm{H})$ PAH $(\mathrm{n}=109)$, CTD-PAH $(\mathrm{n}=111)$ and CTEPH $(\mathrm{n}=131))$ assessed the relationship between emPHasis-10 and survival [11]. In that study, Cox regression analysis demonstrated emPHasis-10 to be predictive of survival, independent of WHO functional class, in congenital heart disease-associated PAH, but not in $(\mathrm{I} / \mathrm{D} / \mathrm{H}) \mathrm{PAH}$ or CTD-PAH. In our study, which included much larger numbers of patients with (I/D/H)PAH and CTD-PAH, emPHasis-10 was an independent prognostic marker in (I/D/H)PAH and CTD-PAH, even when allowing for a number of variables known to be strongly prognostic in $\mathrm{PAH}$ (including mean RAP and cardiac index). This was not the case for WHO functional class and it is interesting to note that BoucLY et al. [14] also observed that baseline WHO functional class was not an independent predictor of outcome in their paper from the French Registry.

In incident patients, an exploratory risk stratification approach separating emPHasis-10 scores into three bands, based on an equal range of scores in each group (thresholds of $\leqslant 16,17-33$ and $\geqslant 34$ ), identified distinct risk groups with significant survival differences (corresponding 1 -year mortality of 5\%, $10 \%$ and $23 \%$, respectively). These levels of 1 -year mortality are very similar to the risk thresholds of low $(<5 \%)$, intermediate $(5-10 \%)$ and high $(>10 \%)$ risk proposed by the European Society of Cardiology (ESC)/European Respiratory Society (ERS) guidelines for risk stratification in PAH. Using these risk thresholds, we determined that patients who either improved to intermediate or low risk at follow-up emPHasis-10 assessment, or deteriorated to high risk, had similar longer-term survival to those patients who were originally in those risk groups. This effect has been seen in a number of other risk stratification parameters and scores [25-27], and the importance of achieving specific PROM thresholds in PAH has also been observed with the generic SF-36 [28]. The majority of patients were in WHO functional class III at the time of diagnosis and we were therefore interested in whether the emPHasis-10 score could refine these patients into higher and lower risk groups. We observed that a threshold score $\geqslant 34$ was indeed able to identify WHO functional class III patients at higher and lower risk of 1-year mortality at both diagnosis and at first follow-up.

We observed moderate correlations between emPHasis-10 and exercise capacity (6MWD, $\mathrm{r}=0.55$; ISWD, $r=0.50)$ and between emPHasis-10 and WHO functional class ( $r=0.50)$, and only weak correlations with pulmonary haemodynamics $(\mathrm{r}=0.17-0.21)$. The correlations between emPHasis-10 and exercise capacity compare favourably with some reports regarding the other two PH-specific PROMs. GomberG-MAITLAND et al. [22] reported weaker correlations between 6MWD and the three domains of CAMPHOR (symptoms $(\mathrm{r}=0.35)$, functioning $(\mathrm{r}=0.45)$ and $\mathrm{QoL}(\mathrm{r}=0.33))$ in 147 PAH patients, while CHIN et al. [24] observed weak to moderate correlation between domains of the PAH-SYMPACT tool and 6MWD ( $\mathrm{r}=-0.14$ to -0.57 ) in $278 \mathrm{PAH}$ patients. More recently however, REIs et al. [29] observed stronger correlation between 6MWD and the three CAMPHOR domains (symptoms $(\mathrm{r}=-0.67)$, functioning $(\mathrm{r}=-0.74)$ and QoL $(\mathrm{r}=-0.61)$ ) in 49 patients with $\mathrm{PAH}$ or CTEPH. To date, there have been no reports of correlation of $\mathrm{PH}$-specific PROMs with pulmonary haemodynamics. The correlations we observed were, however, comparable to those observed by MATHAi et al. [18] between components of the generic SF-36 HRQoL tool and haemodynamics in 87 patients with $\mathrm{PAH}$ (although, in their study, many of these correlations were non-significant).

Finally, we have demonstrated that an improvement in emPHasis-10 score at follow-up of at least the MDC (nine or greater) was associated with an increase in exercise capacity in incident patients, whereas a reduction in emPHasis-10 score by nine or greater was associated with a decrease in exercise capacity when assessed by the ISWD. The vast majority of incident patients will have been started on PAH therapies, whereas in prevalent patients there may have been no treatment change between assessments, which may partly explain the stronger relationship between change in walk distance and change in emPHasis-10 score in the incident group. The reason for the stronger relationship between change in ISWD (as opposed to 6MWD) and change in emPHasis-10 is not clear, but may reflect the different nature of the tests (the 
ISWT is an externally-paced measure of maximal exercise capacity while the 6MWT is an internally-paced assessment of sub-maximal exercise capacity). These data suggest that emPHasis-10 is responsive to change; however, further work is needed to define the minimal clinically important difference.

\section{Limitations}

While this study was able to demonstrate important associations between emPHasis-10 score and time to death or transplantation, other measures of clinical deterioration including hospitalisation due to heart failure and escalation of therapy were unavailable. In addition, while emPHasis-10 scores were prospectively collected, this was a retrospective study and there were some data availability issues. Treatment data were not available and it is possible that PAH-specific therapies might affect HRQoL both negatively (in terms of side effects and the effects of complex treatments on lifestyle) and also positively (in terms of improvements in right-ventricular function translating into amelioration of symptoms). Finally, data regarding comorbidities, such as the presence and extent of parenchymal lung disease in patients with CTD-PAH, were unavailable. Assuming that comorbidities such as lung disease adversely affect HRQoL, the inclusion of patients with parenchymal lung disease would likely weaken the relationships between emPHasis-10 and functional parameters, treatment response and survival.

\section{Conclusion}

The emPHasis-10 score correlates with WHO functional class, exercise capacity and haemodynamics, and is an independent prognostic marker in patients with $(\mathrm{I} / \mathrm{D} / \mathrm{H}) \mathrm{PAH}$ and CTD-PAH. It has utility in risk stratification in addition to currently used parameters. The survival of patients within WHO functional class III can be further stratified using emPHasis-10 score. Improvement in emPHasis-10 is associated with improvement in exercise capacity, although further work to determine the minimal clinically important difference is required.

Acknowledgements: The authors would like to thank the following for their assistance in managing local databases: Jay Thacker (Golden Jubilee Hospital); George Villa (Hammersmith Hospital); Stuart Craig, Carl Harries and Della Stokes (Royal Brompton Hospital); Sheila Forshaw, Neil Hamilton, Amanda Haythorne, Amanda Poirrier and Daniel Roberts (Royal Hallamshire Hospital); Nisha Abraham, Samantha Ali, Natalie Grout, Chindu John and Nicola Speed (Royal Papworth Hospital).

Conflict of interest: R.A. Lewis reports non-financial support for meeting attendance from Actelion Pharmaceuticals, outside the submitted work. I. Armstrong has nothing to disclose. C. Bergbaum has nothing to disclose. M.J. Brewis has nothing to disclose. J. Cannon has nothing to disclose. A. Charalampopoulos reports grants, personal fees and non-financial support from Actelion Pharmaceuticals, personal fees and non-financial support from Novartis, and grants from Bayer and GSK, outside the submitted work. A.C. Church has nothing to disclose. J.G. Coghlan has nothing to disclose. R.J. Davies has nothing to disclose. K. Dimopoulos has nothing to disclose. C. Elliot reports personal fees for advisory board work and lectures from Actelion Pharmaceuticals, GlaxoSmithKline and Bayer, as well as grants from Pfizer, Actelion Pharmaceuticals and Bayer, outside the submitted work. J.S.R. Gibbs reports personal fees from Acceleron, Arena, Bayer, Complexa, Pfizer and GSK, as well as grants and personal fees from Actelion and United Therapeutics, outside the submitted work. W. Gin-Sing has nothing to disclose. G. Haji has nothing to disclose. A.G. Hameed has nothing to disclose. L.S. Howard has nothing to disclose. M.K. Johnson reports grants and personal fees for meeting attendance, lectures and advisory board work from Actelion and MSD, outside the submitted work. A. Kempny has nothing to disclose. D.G. Kiely reports grants, personal fees and non-financial support from Actelion, Bayer and GSK, as well as personal fees and non-financial support from MSD, outside the submitted work. F. Lo Giudice has nothing to disclose. C. McCabe has nothing to disclose. A.J. Peacock has nothing to disclose. O. Peleyeju has nothing to disclose. J. Pepke-Zaba has nothing to disclose. G. Polwarth has nothing to disclose. L. Price reports educational grants from Actelion J\&J, during the conduct of the study. I. Sabroe reports grants and personal fees for advisory board work from AstraZeneca, as well as grants from GSK, outside the submitted work. B.E. Schreiber has nothing to disclose. K. Sheares reports educational support from Actelion, Bayer and GSK, as well as personal fees for lectures and consultancy from Actelion, outside the submitted work. D. Taboada reports fees for lectures and education and travel grants from Actelion, Bayer, GlaxoSmithKline, Lilly, MDS and Pfizer, outside the submitted work. A.A.R. Thompson reports grants from the British Heart Foundation (Intermediate Clinical Fellowship FS/18/13/3328), during the conduct of the study, as well as non-financial support for travel to meetings from Actelion Pharmaceuticals Ltd, outside the submitted work. M.R. Toshner has nothing to disclose. I. Wanjiku has nothing to disclose. S.J. Wort has nothing to disclose. J. Yorke has nothing to disclose. R. Condliffe reports grants, personal fees and non-financial support from Actelion Pharmaceuticals and Bayer, as well as grants from GSK, outside the submitted work.

Support statement: A.A.R. Thompson is supported by a British Heart Foundation Intermediate Clinical Fellowship (FS/ 18/13/3328). Funding information for this article has been deposited with the Crossref Funder Registry.

\section{References}

$1 \quad$ Kiely DG, Elliot CA, Sabroe I, et al. Pulmonary hypertension: diagnosis and management. BMJ 2013; 346 : f2028.

2 Dumitrescu D, Sitbon O, Weatherald J, et al. Exertional dyspnoea in pulmonary arterial hypertension. Eur Respir Rev 2017; 26: 170039.

3 Lau EM, Humbert M, Celermajer DS. Early detection of pulmonary arterial hypertension. Nat Rev Cardiol 2015; 12: $143-155$.

4 Condliffe R. Living with pulmonary hypertension: quality not just quantity. Eur Respir J 2011; 38: 512-513. 
5 Sitbon O, Gomberg-Maitland M, Granton J, et al. Clinical trial design and new therapies for pulmonary arterial hypertension. Eur Respir J 2019; 53: 1801908.

6 McKenna SP, Doughty N, Meads DM, et al. The Cambridge Pulmonary Hypertension Outcome Review (CAMPHOR): a measure of health-related quality of life and quality of life for patients with pulmonary hypertension. Qual Life Res 2006; 15: 103-115.

7 Yorke J, Corris P, Gaine S, et al. emPHasis-10: development of a health-related quality of life measure in pulmonary hypertension. Eur Respir J 2014; 43: 1106-1113.

8 McCollister D, Shaffer S, Badesch DB, et al. Development of the Pulmonary Arterial Hypertension-Symptoms and Impact (PAH-SYMPACT ${ }^{*}$ ) questionnaire: a new patient-reported outcome instrument for PAH. Respir Res 2016; 17: 72.

9 Foster E, Guillen A, Lara K, et al. Linguistic validation of the emPHasis-10 questionnaire: a patient-reported outcome instrument for assessing QoL in pulmonary hypertension (PH). Value in Health 2015; 18: A744.

10 Odevoglu P, Demir R, Okumus G, et al. Evaluation of the validity and reliability of the Turkish translation of the emPHasis-10 questionnaire in patients with pulmonary hypertension. ERS International Congress, Paris, 2018.

11 Favoccia C, Kempny A, Yorke J, et al. EmPHasis-10 score for the assessment of quality of life in various types of pulmonary hypertension and its relation to outcome. Eur J Prev Cardiol 2019; 26: 1338-1340.

12 Benza RL, Gomberg-Maitland M, Elliott CG, et al. Predicting survival in patients with pulmonary arterial hypertension: the REVEAL Risk Score Calculator 2.0 and comparison with ESC/ERS-based risk assessment strategies. Chest 2019; 156: 323-337.

13 Galiè N, Humbert M, Vachiery J-L, et al. 2015 ESC/ERS guidelines for the diagnosis and treatment of pulmonary hypertension. Eur Respir J 2015; 46: 903.

14 Boucly A, Weatherald J, Savale L, et al. Risk assessment, prognosis and guideline implementation in pulmonary arterial hypertension. Eur Respir J 2017; 50: 1700889.

15 National audit of pulmonary hypertension, 10th annual report. NHS Digital, 2019. https://digital.nhs.uk/ data-and-information/publications/statistical/national-pulmonary-hypertension-audit/2019 Date last updated: October 24, 2019. Date last accessed: July 31, 2020.

16 Simonneau G, Montani D, Celermajer DS, et al. Haemodynamic definitions and updated clinical classification of pulmonary hypertension. Eur Respir J 2019; 53: 1801913.

17 de Vet HC, Terwee CB, Ostelo RW, et al. Minimal changes in health status questionnaires: distinction between minimally detectable change and minimally important change. Health Qual Life Outcomes 2006; 4: 54.

18 Mathai SC, Suber T, Khair RM, et al. Health-related quality of life and survival in pulmonary arterial hypertension. Ann Am Thorac Soc 2016; 13: 31-39.

19 McCabe C, Bennett M, Doughty N, et al. Patient-reported outcomes assessed by the CAMPHOR questionnaire predict clinical deterioration in idiopathic pulmonary arterial hypertension and chronic thromboembolic pulmonary hypertension. Chest 2013; 144: 522-530.

20 Cenedese E, Speich R, Dorschner L, et al. Measurement of quality of life in pulmonary hypertension and its significance. Eur Respir J 2006; 28: 808-815.

21 Meads DM, McKenna SP, Doughty N, et al. The responsiveness and validity of the CAMPHOR Utility Index. Eur Respir J 2008; 32: 1513-1519.

22 Gomberg-Maitland M, Thenappan T, Rizvi K, et al. United States validation of the Cambridge Pulmonary Hypertension Outcome Review (CAMPHOR). J Heart Lung Transplant 2008; 27: 124-130.

23 Delcroix M, Howard L. Pulmonary arterial hypertension: the burden of disease and impact on quality of life. Eur Respir Rev 2015; 24: 621-629.

24 Chin KM, Gomberg-Maitland M, Channick RN, et al. Psychometric validation of the Pulmonary Arterial Hypertension-Symptoms and Impact (PAH-SYMPACT) questionnaire: results of the SYMPHONY Trial. Chest 2018; 154: 848-861.

25 Hoeper MM, Kramer T, Pan Z, et al. Mortality in pulmonary arterial hypertension: prediction by the 2015 European pulmonary hypertension guidelines risk stratification model. Eur Respir J 2017; 50: 1700740.

26 Kylhammar D, Kjellstrom B, Hjalmarsson C, et al. A comprehensive risk stratification at early follow-up determines prognosis in pulmonary arterial hypertension. Eur Heart J 2018; 39: 4175-4181.

27 Lewis RA, Johns CS, Cogliano M, et al. Identification of cardiac magnetic resonance imaging thresholds for risk stratification in pulmonary arterial hypertension. Am J Respir Crit Care Med 2020; 201: 458-468.

28 Fernandes CJCS, Martins BCS, Jardim CVP, et al. Quality of life as a prognostic marker in pulmonary arterial hypertension. Health Qual Life Outcomes 2014; 12: 130.

29 Reis A, Santos M, Vicente M, et al. Health-related quality ofl in pulmonary hypertension and its clinical correlates: a cross-sectional study. Biomed Res Int 2018; 2018: 3924517. 